\section{Neuro-psychiatric Effects of COVID-19}

Sir,

The pandemic of severe acute respiratory syndrome coronavirus 2 (SARS-CoV-2), also known as coronavirus disease 2019 (COVID-19), has triggered grave threats to physical health; and has endangered the life of people all over the world. Along with serious systemic and pathophysiological consequences, the pandemic has also become a source of many direct or indirect psychological illnesses like, anxiety, depression and panic disorder, which ultimately lead to an increase in the global mental health burden. ${ }^{1}$ In China, the epidemic has caused $16.5 \%$ of moderate to severe depressive symptoms; $28.8 \%$ of moderate to severe anxiety symptoms; and $8.1 \%$ of moderate to severe stress in the overall population along with sleep disturbances. ${ }^{2}$ The continuously evolving disease and associated risk factors have led to various psychological stresses including coronaphobia, which is exaggerated-triggered fear of contracting COVID-19, that can cause excessive stress about physiological symptoms, personal as well as occupational loss and raised safety seeking behaviours, which eventually impair the overall daily living functions of an individual. ${ }^{3}$

The sufferers of COVID-19 may present with the symptoms of neurological ailments, such as, headache, altered conscious status and abnormal tingling sensations. Severity of symptoms depends on the overall severity of the disease. A number of patients affected by this disease reported loss of the senses of smell and taste, so it is likely to observe anosmia and dysgeusia in subjects suffering from COVID-19. Brain tissue edema along with partial neurological degeneration was also observed and cases of viral encephalitis have also been reported. Presence of this virus in cerebrospinal fluid (CSF) has been confirmed by the researchers, which also signifies the damage of nervous system. The possible mechanisms of nervous system injury by this virus are direct infection, circulatory dissemination, neuronal pathway, hypoxic injury, and immune injury. The virus can directly involve the neurological system and induce infection, as is evident by the presence of virus in CSF of some patients. ${ }^{4}$ Neuronal pathways are a major route for viruses to enter the nervous system as they typically infect the motor as well as sensory nerves and can travel through the nerve fibers by attaining anterograde or retrograde transportation via motor proteins, dynein and kinesins. The virus can reach the brain and CSF by neuronal pathway of olfaction within seven days and can cause injury. Thus, the virus can attack the brain through multiple pathways. ${ }^{5}$ Another mechanism is hypoxic injury; as the lung tissue is invaded, it leads to interstitial lung damage, gas exchange disturbance and ultimately brain hypoxia which causes dilation of the cere- bral blood vessels that leads to inflammation, edema and impediment of cerebral perfusion. Moreover, when the immune system responds, it can lead to severe brain damage. ${ }^{4}$ All of these neuro-psychiatric effects have also been observed in patients of COVID-19 as it may infect brain or activate an immune response leading to severe effects on mental health aswell asfunctioning of brain. ${ }^{4}$

COVID-19 may involve nervous system and the extent of involvement depends upon the immune mechanism and response of host. The patients suffering from this virus can have symptoms of neurological system and may present with psychiatric disturbances. Therefore, it is necessary for healthcare professionals to evaluate the coronavirus infected patients for neuro-psychiatric symptoms, which can be ignored in the process of saving the lives of persons affected by the deadly virus. Early awareness and analysis in this regard may delay the complications and improve the prognosis.

\section{CONFLICT OF INTEREST:}

The authors declared no conflict of interest.

\section{AUTHORS' CONTRIBUTION:}

KK: Concept, design, and accountable for all the work.

NS: Drafting, revision, and accountable for all the work.

MMAA: Critical analysis, final approval, and accountable for all the work.

\section{REFERENCES}

1. Qiu J, Shen B, Zhao M, Wang Z, Xie B, Xu Y. A nationwide survey of psychological distress among Chinese people in the COVID-19 epidemic: implications and policy recommendations. General psychiatry 2020; 33(2): e100213. doi: 10.1136/gpsych-2020-100213.

2. Rajkumar RP. COVID-19 and mental health: A review of the existing literature. Asian J Psychiatr 2020; 102066. doi: 10.1016/j.ajp.2020.102066.

3. Arora A, Jha AK, Alat P, Das SS. Understanding coronaphobia. Asian J Psychiatr 2020; 54:102384. doi.org/10. 1016/j.ajp.2020.102384.

4. Wu Y, Xu X, Chen Z, Duan J, Hashimoto K, Yang L, et al. Nervous system involvement after infection with COVID-19 and other coronaviruses. Brain Behav Immun 2020; 87:18-22. doi: 10.1016/j.bbi.2020.03.031.

5. Swanson II PA, McGavern DB. Viral diseases of the central nervous system. Curr Opin Virol 2015; 11:44-54. doi.org/ 10.1016/j. coviro.2014.12.009.

Kiran Khushnood ${ }^{1}$, Nasir Sultan ${ }^{2}$ and Malik Muhammad Ali Awan ${ }^{1}$

${ }^{1}$ Department of Rehabilitation Sciences, Isra University, Islamabad, Pakistan

${ }^{2}$ Department of Rehabilitation Sciences, Shifa Tameer-e-Millat University, Islamabad, Pakistan 
Correspondence to: Dr. Malik Muhammad Ali Awan, Department of Rehabilitation Sciences, Isra University, Islamabad, Pakistan

E-mail:mmaawan@gmail.com
Received: September 16, 2020; Revised: September 18, 2020;

Accepted: September 23, 2020

DOI: https://doi.org/10.29271/jcpsp.2020.JCPSPCR.CR181 